\section{$\underset{\substack{\text { hommes } \\ \text { \& migrations }}}{ }$}

\section{Hommes \& migrations}

Revue française de référence sur les dynamiques

migratoires

$1314 \mid 2016$

Migrations chinoises et générations

\title{
Pourquoi en finir avec l'exil ?
}

Entretien avec Alexis Nouss

Marie Poinsot et Nicolas Treiber

\section{(2) OpenEdition \\ 1 Journals}

Édition électronique

URL : http://journals.openedition.org/hommesmigrations/3648

DOI : 10.4000/hommesmigrations.3648

ISSN : 2262-3353

Éditeur

Musée national de l'histoire de l'immigration

Édition imprimée

Date de publication : 1 avril 2016

Pagination : 116-119

ISBN : 978-2-919040-35-3

ISSN : 1142-852X

Référence électronique

Marie Poinsot et Nicolas Treiber, « Pourquoi en finir avec l'exil ? », Hommes \& migrations [En ligne],

1314 | 2016, mis en ligne le 19 septembre 2016, consulté le 15 septembre 2020. URL : http://

journals.openedition.org/hommesmigrations/3648 


\section{SPÉCIAL FRONTIÈRES}

\section{POURQUOI EN FINIR AVEC L'EXIL ?}

Entretien avec ALEXIS NOUSS, auteur de La Condition de l'exilé. Penser les migrations contemporaines, Paris, éd. de la MSH, 2015.

Réalisé par MARIE POINSOT et NICOLAS TREIBER.

Hommes \& Migrations : Pourquoi avoir privilégié cette notion d'exil pour aborder les réalités des migrations contemporaines?

Alexis Nouss : Les termes "réfugié" ou "migrant" qui se partagent les discours actuels dépendent de régimes d'analyse précis : le migrant désigne une réalité socioéconomique, le réfugié, une réalité juridique. Si ces grilles d'analyse très rigides fondées sur le social, l'économique et le juridique rassurent, elles tendent à effacer l'expérience humaine de tous ces "arrivants". Considérer ces derniers comme des exilés, c'est pouvoir les accueillir également dans notre imaginaire. L'exil renvoie à une expérience anthropologique fondamentale qui devrait inspirer les politiques dans la mesure où elle questionne les critères mêmes sur lesquels est fondée la gestion des migrations : la notion de nationalité et la notion de territoire.

H\&M : Du point de vue littéraire, le terme d'“exil" revient souvent pour mettre l'accent sur une certaine expérience de la mobilité.

A. N. : L'expérience exilique dessine un arc qui soutient l'histoire de la littérature occidentale. À l'aube hellène, L'Odyssée d'Homère, l'un des premiers récits fondateurs de la littérature occidentale. Ulysse n'est pas seulement un voyageur, c'est un exilé qui, revenu à Ithaque, ne pense qu'à repartir. À l'autre bout, dans la modernité,
l'Ulysse de James Joyce. De l'antiquité à la modernité, on retrouve la même figure. C'est également le cas avec Ovide qui écrit Les Tristes alors qu'il est exilé sur les rivages de la Mer rouge et, dans les années 1920, Ossip Mandelstam publiant un recueil de poèmes intitulé Tristia en hommage au premier. Ovide, toujours, avec ses Métamorphoses puis Kafka et sa Métamorphose - dans les deux cas, des exils de la condition humaine. Prenons Victor Hugo, notre grand poète national français, qui vécut vingt ans en exil, près d'un quart de sa vie. II y écrivit certaines des œuvres phares de sa production comme Les Misérables ou Les Contemplations. On peut relire tout Les Misérables au prisme de l'exil, son incipit contant un retour d'exil avec l'arrivée de Jean Valjean du bagne de Cayenne, un double exil, géographique et social. Expérience d'exclusion qui frappe tous les autres personnages, de Cosette à Gavroche.

\section{$H \& M$ : Ces récits créés en exil sont-ils des récits fondateurs de la littérature et de la pensée contemporaine?}

A. N. : Exactement. Et puis n'oublions pas les textes des monothéismes, traversés par l'expérience exilique. Dans l'Ancien testament, l'exode des Hébreux en Égypte. Dans le Nouveau testament, la Sainte Famille en Égypte. Dans le Coran c'est, évidemment, l'Hégire, Mahomet partant de la Mecque pour aller à Médine. L'exil figure dans 
I'ADN culturel des sociétés occidentales. Le fait de nommer "exilé" ceux qui arrivent d'Érythrée, d'Afghanistan, de Syrie, etc., permet de les mettre en rapport avec cette mémoire exilique que porte la littérature occidentale. Alors que je ne peux qualifier décemment Victor Hugo ou Ovide de "migrants", appeler les uns et les autres "exilés" permet de faire ressortir ce qu'ils ont en commun, ce que j'appelle dans mon ouvrage "I'exiliance". En utilisant la littérature comme une espèce de réceptacle de la mémoire exilique et au nom de cette mémoire-là, je peux bâtir autour de cette "exiliance" une sensibilité permettant d'accueillir celui ou celle qui arrive. Je me détourne de la simple perspective socio-économique ou juridique, pour non pas la nier, mais trouver une autre source d'inspiration et la nourrir avec le matériau de la littérature. C'est aussi ce que vous faites au Palais de la Porte Dorée, même si je crains qu'on ne fasse pas le lien entre ces destins mis de l'avant et ceux et celles qui arrivent aujourd'hui.

$H \& M$ : Les discours patrimoniaux et littéraires ne risquent-ils pas de manquer leur cible, leurs effets étant condamnés à demeurer infimes face à la force de frappe des médias et des discours politiques sur l'opinion et les imaginaires?

A. N. : C'est pourtant le seul moyen. En continuant de raisonner en termes de citoyenneté et de territorialité, les deux principes mis à l'épreuve par l'expérience de la migration, on demeure dans l'impasse. Pour changer de paradigme, il faut changer d'inspiration, reconnaître l'exilé derrière le migrant et l'accueillir en tant que tel. Or celle-ci est là, dans le musée, dans la littérature, dans les récits fondateurs du monothéisme. L'Europe a réussi à exister en fonction d'une certaine mémoire européenne. Ceux qui ont fondé la communauté européenne au lendemain de la guerre l'ont fait au nom cette mémoire héritée du XVIII siècle et forgée antérieurement. De la même manière, nous devons agir par rapport aux migrations en fonction de notre mémoire exilique. Notre rôle, vous au musée, nous dans les universités, c'est d'activer cette mémoire-là.

H\&M : Vous définissez une notion de l'exil plus vaste que la seule expérience de la mobilité. On oublie souvent que les exilés viennent de quelque part et qu'ils maintiennent une relation avec ce lieu. En quoi ne peut-on pas couper l'exilé du lien avec son pays d'origine?

A. N. : L'exil est une expérience bipolaire, dans un sens positif. L'exilé est celui qui vit dans un nouveau présent sans abandonner le présent de son passé. Une fois arrivé, il doit se bâtir un nouveau vécu. Le seul moyen dont il dispose pour se forger des dispositifs lui permettant de s'installer dans un présent qu'il ne maîtrise pas est de se tourner vers son passé. Le pays, la langue, la culture et le ciel quittés sont le terreau dans lequel va puiser l'exilé. Il est totalement vain de croire qu'il puisse brutalement passer dans une nouvelle réalité L'être humain est un être de devenir et non pas de la transformation brutale. Continuité essentielle et constituante de l'expérience exilique l'exilé est d'emblée multiculturel, offrant à ce titre une exemplarité politique dont nos sociétés ont fort besoin. II a une première et une deuxième vie et les deux communiquent. Sa nostalgie l'aide au présent à se forger un nouvel être. II n'y a pas de césure. Au contraire, il faut accueillir l'arrivant avec son passé et ce qu'il a quitté D'une certaine manière, le territoire quitté voyage avec l'exilé...

H\&M : Vous introduisez aussi la notion d'une expérience de l'exil non vécu en pointant ses effets identitaires sur les jeunes nés de parents exilés sans l'avoir connu eux-mêmes. La non reconnaissance de cette transmission de l'exil d'une génération à l'autre pourraitelle expliquer les lignes de fractures et les tensions au sein des sociétés européennes ?

A. N. : Cela ne fait aucun doute. Je renvoie à la grammaire : comme participe présent, le mot 


\section{SPÉCIAL FRONTIÈRES}

"migrant" est attaché à l'action d'un sujet en train d'être effectuée. Quand l'action prend fin, la définition s'efface et devient obsolète, elle ne peut pas être transmise. Parler "d'exiliance" permet de distinguer une condition et une conscience, pouvant coïncider ou non. On peut vivre dans une condition d'exil sans ressentir la conscience de l'exil. Au contraire, on peut posséder une conscience de l'exil sans en éprouver la condition. Je crois que c'est précisément le cas des générations qui se sont éveillées en exprimant un manque identitaire. Cela me semble pertinent comme grille d'analyse pour un certain nombre de problématiques sociétales actuelles. II se pose également un problème d'étymologie. Jusqu'au XIXe siècle, l'étymologie voulait que le mot "exil" vienne de "ex solus", c'est-à-dire "parti d'un sol". Or la deuxième syllabe du mot renvoie à "salire" qui veut dire "sauter". Ex-salire signifie le "saut vers". L'expérience de l'exil comprend à la fois du passé et du futur. Un avenir conjoint dans l'exil qui peut faire l'objet d'une transmission.

\section{H\&M : Comment expliquez-vous l'engoue- ment pour le thème de l'exil dans la littérature contemporaine de langue française?}

A. N. : Il n'est pas si nouveau. Je vous donne trois noms, dont deux prix Nobel, Marguerite Duras, Patrick Modiano, J. M. G. Le Clezio. Ces trois auteurs majeurs et même emblématiques de la modernité française de la deuxième moitié du XXe siècle ont placé l'exil au cœur de leurs interrogations. Dans le cas de Duras, il est nourri biographiquement. Tous ses personnages affichent un exil identitaire, ils ne sont jamais vraiment eux-mêmes; il y a toujours un ailleurs qui les hante ou vers lequel ils vont. L'espace même de l'imaginaire de Modiano, le Paris de la collaboration, de l'occupation, est un Paris des exilés. Ses personnages ont des identités qui ne correspondent pas à leur carte d'identité. Comme s'ils avaient un accent étranger. Quant à Le Clézio, il a choisi la scène du monde. Dans l'un de ses plus grands ouvrages, Désert, les personnages sont définis par une non-identité territoriale qui les pousse à choisir l'exil comme résidence.

Duras, Modiano, Le Clézio constituent une première strate - non-chronologique - de la scène littéraire française de l'après-guerre. Puis s'y ajoute l'ouverture à la francophonie. Les auteurs maghébins, africains, antillais ont apporté leurs expériences exiliques dans leur rapport même à la culture et à la langue française, comme Mohammed Dib ou Édouard Glissant. Une troisième strate serait enfin composée par des auteurs contemporains, de Tahar Ben Jelloun à Laurent Gaudé. Appuyés sur les deux premières strates, ils font leur travail d'écrivains en donnant voix à ce que laisse échapper le discours commun à propos de l'expérience exilique. La littérature française que l'on accuse de nombrilisme, de narcissisme, est ici à la hauteur de sa fonction testimoniale.

\section{H\&M : Montrer des aventures humaines aux marges de la société, aux marges du récit natio- nal permet-il d'échapper à ce nombrilisme ?}

A. N. : La littérature expose et entretient le souci de l'autre, le souci de l'accueillir dans un processus de construction identitaire. C'est pourquoi la littérature aura toujours besoin de héros, non pas au sens épique mais comme modèle perceptif. Le héros serait celui qui vient déranger notre tranquillité. Ainsi, les personnages de Laurent Gaudé, de Mathias Enard, de Marie N'Daye, apportent une altérité dont la littérature a besoin. Pourquoi commencer à écrire sinon pour explorer une altérité ? Et pourquoi lire, sinon afin de poursuivre cette exploration ? La littérature française peut et doit être à la hauteur de cette fonction, éminemment politique dans le sens du vivre ensemble. La littérature nous apprend l'autre, tout simplement, par exemple l'arrivant, 
l'exilé. En outre, l'actualité a mis en lumière l'importance du récit dans le destin du sujet migrant contemporain. À deux titres : l'arrivant, s'il veut être admis comme réfugié, doit fournir un récit de son parcours qui satisfera aux conditions d'octroi du statut, au point que certains scenarii sont préférés parce qu'ils ont fait preuve d'efficacité. Par ailleurs, on constate, recueillis sur des sites Internet ou sur papier, une prolifération de récits de vie comme si les exilés, dépouillés de leur appartenance sociale, éprouvaient le besoin de se reconstituer une identité en racontant leur histoire. L'exil contemporain sacrifie aussi à un régime narratif.

\section{H\&M : En quoi la langue française s'enrichit- elle de l'apport de ces auteurs qui l'ont adop- tée pour écrire leur exil ? Trouve-t-on une incidence entre le thème et le traitement lit- téraire de l'exil ?}

A. N. : Oui, chez certains écrivains. Je prendrai deux exemples d'exilés inscrits dans la littérature française au point d'avoir obtenu un siège à l'Académie française : Dany Laferrière et Andreï Makine. Dany Laferrière, capable de grande sobriété, joue parfois d'une phrase et d'une structure virevoltante, un ludisme - il le revendique lui-même - propre à l'écrivain des Caraïbes. Non pas dans le sens d'une provenance car c'est un écrivain de l'exil. Le premier, celui d'Haïti, n'était pas volontaire mais par la suite - Montréal, Miami, la France -, Laferrière a mis en place un mouvement perpétuel que prolonge son écriture. Quant à Andreï Makine, ne trouvant pas d'éditeur en France, il a prétendu que ses écrits étaient des traductions du russe. Or, on retrouve chez lui une retenue, un classicisme de la phrase qui rappelle les réserves du traducteur à introduire trop d'étrangeté dans sa traduction. Quelques brefs exemples stylistiques qui pourraient formellement aider à décrire une littérature de l'exil.

\section{H\&M : Peut-on en finir avec l'exil ?}

A. N. : Je pense l'exil non pas comme une identité mais comme une condition. J'essaie de penser une condition exilique comme il y a une condition féminine, noire, juive. Ce sont des catégories anthropologiques fondamentales, des modes de traduction d'une condition qui n'est jamais accessible en tant que telle la condition humaine. Or l'être humain est un être d'exil.

L'histoire de l'humanité commence par des communautés, des peuplades qui quittent un territoire, traversent des milliers de kilomètres pour s'installer dans un autre. Le territoire européen lui-même est façonné par des exils. Ce sont les Celtes, les Francs, les Romains... des populations entières qui se sont installées, ont prospéré et créé des cultures dans des endroits où elles ne sont pas nées.

En contrepoint, il y a la phase sombre de cette pulsion de l'Europe à vouloir s'installer ailleurs, à savoir le colonialisme. Ainsi, la condition exilique peut nous mettre en crise, interroger notre chez-soi et notre quant-à-soi, pour emprunter à Lévinas, et motiver notre volonté d'y échapper. Ce n'est pas une identité fixe. Une condition sera un support identitaire, un catalyseur de subjectivation, c'est-à-dire un répertoire de sensations, d'idées, de concepts ou d'analyses dont la cohérence est liée à une expérience. En d'autres termes, une condition est la traduction en culture, en pensée, en mémoire d'une expérience.

L'expérience de l'exil existe pour chaque être humain depuis l'aube de l'humanité et pour chacun depuis sa propre genèse - nous sommes tous exilés d'un ventre maternel. C'est pourquoi on ne doit pas vouloir en finir avec l'exil mais, au contraire, l'installer au cœur même de nos dispositifs de pensée, en reconnaissant dans la condition exilique une possibilité fondamentale du devenir humain. 